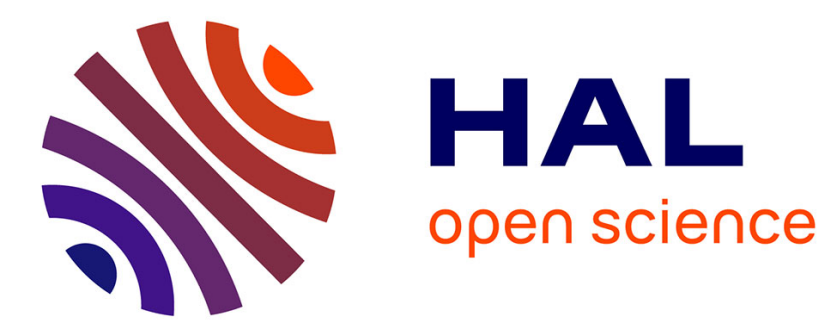

\title{
Perturbation Analysis for Robust Damage Detection with Application to Multifunctional Aircraft Structures
}

Rafik Hajrya, Nazih Mechbal

\section{To cite this version:}

Rafik Hajrya, Nazih Mechbal. Perturbation Analysis for Robust Damage Detection with Application to Multifunctional Aircraft Structures. 2015. hal-01100545

\section{HAL Id: hal-01100545 \\ https://hal.science/hal-01100545}

Preprint submitted on 12 Jan 2015

HAL is a multi-disciplinary open access archive for the deposit and dissemination of scientific research documents, whether they are published or not. The documents may come from teaching and research institutions in France or abroad, or from public or private research centers.
L'archive ouverte pluridisciplinaire HAL, est destinée au dépôt et à la diffusion de documents scientifiques de niveau recherche, publiés ou non, émanant des établissements d'enseignement et de recherche français ou étrangers, des laboratoires publics ou privés. 


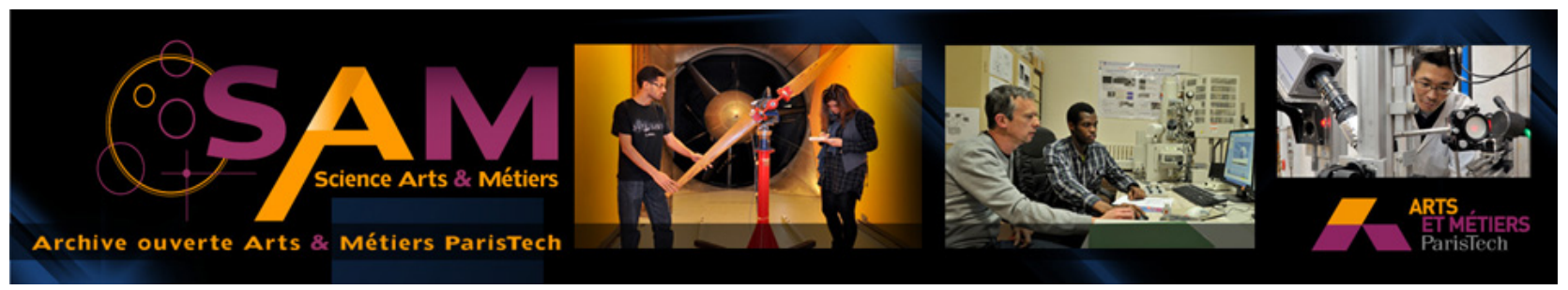

Science Arts \& Métiers (SAM)

is an open access repository that collects the work of Arts et Métiers ParisTech researchers and makes it freely available over the web where possible.

This is an author-deposited version published in: http://sam.ensam.eu

Handle ID: .http://hdl.handle.net/10985/9174

\section{To cite this version :}

Rafik HAJRYA, Nazih MECHBAL - Perturbation Analysis for Robust Damage Detection with Application to Multifunctional Aircraft Structures - Smart Structures and Systems, An International Journal - Vol. XXX, nXXX, p.XXX - 2015 


\title{
Perturbation Analysis for Robust Damage Detection with Application to Multifunctional Aircraft Structures
}

\author{
Rafik Hajrya ${ }^{* a}$ and Nazih Mechbal ${ }^{*}$ \\ Arts et Métiers ParisTech (ENSAM), Process and Engineering in Mechanics and Materials Laboratory (PIMM) \\ CNRS-UMR 8006-151 Boulevard de l'hôpital, 75013, Paris, France
}

\begin{abstract}
The most widely known form of multifunctional aircraft structure is smart structures for structural health monitoring (SHM). The aim is to provide automated systems whose purposes are to identify and to characterize possible damage within structures by using a network of actuators and sensors. Unfortunately, environmental and operational variability render many of the proposed damage detection methods difficult to successfully be applied. In this paper, an original robust damage detection approach using output-only vibration data is proposed. It is based on independent component analysis and matrix perturbation analysis, where an analytical threshold is proposed to get rid of statistical assumptions usually performed in damage detection approach. The effectiveness of the proposed SHM method is demonstrated numerically using finite element simulations and experimentally through a conformal load-bearing antenna structure and composite plates instrumented with piezoelectric ceramic materials.
\end{abstract}

Keywords: Structural health monitoring; robust damage detection; decision-making; analytical threshold; matrix perturbation theory; range subspaces; independent component analysis; piezoelectric ceramic material; composite structures; conformal load-bearing antenna structure; impact damage

\section{Introduction}

Ongoing research and development programs on advanced smart structures, particularly for aircraft industries, have come up with the concept of multifunctional aircraft structure (MAS). The principle is to take the advantage of new materials to integrate airframe structure with functional systems. The structure has the ability to respond to changes due to environmental conditions and to perform a number of tasks such as transmit/receive function, structural enhancement and repair (Wang and $\mathrm{Wu}$ 2012), conformal load-bearing antenna structure (CLAS) ( Lockyer et al. 1996) and structural health monitoring (SHM) (Mahzan et al. 2010). On MAS, SHM is the most form of smartness that is studied. It is a broad field encompassing many synergetic technologies that provide together automated systems whose purposes are to identify and characterize possible damage within structures. The SHM problem has occupied many scientific communities in the last

\footnotetext{
*a Ph.D., E-mail: rafik.hajrya@ensam.eu

*b Associate Professor., Email: nazih.mechbal@ensam.eu
} 
decades, and has led to a variety of efficient methods to detect, locate (Liu et al. 2012), classify damages and estimate the remaining useful life (Chang et al. 2007; Farrar and Lieven 2007).

Recently, attention has been paid in damage detection for techniques that exploit the spatial information's collected by the sensors of a monitored structure. These techniques are referred as multivariate analysis and the blind source separation (BSS) family is one of them. The idea of BSS is to separate the response of a number of "source signals" at a number of measurement points through static/convolutive or linear/nonlinear model mixture (Fig. 1), with no direct knowledge about the sources, but based solely on the assumption that the different sources are statistically independent (Comon and Jutten 2010). This problem has given rise to variety of approaches such as the Independent Component Analysis (ICA) (Hyvärinen et al. 2001), Second-Order Blind Identification (SOBI) (Belouchrani et al. 1997).

The importance of BSS in the structural mechanical analysis has been brought up by some precursory works. These later have addressed the relation between the linear normal mode (LNM) identification and BSS techniques as SOBI (McNeill and Zimmerman 2008) and ICA methods (Kerschen et al. 2007). The related works have put in evidence that the modal coordinates are a specific case of sources that have a certain time structure. Thereby, the BSS techniques can be seen as non-parametric output-only modal identification. Furthermore, as damage can produce changes in the modal coordinates, it is then evident that the use of BSS techniques as ICA is an approach to be investigated for damage detection. In that sense, Zang et al. (2004) have presented a damage detection approach based on combining ICA and artificial neural networks (ANN), the authors have used a mixing matrix extracted from an ICA algorithm to train the ANN. Elseifi (2010) has used the sources extracted from ICA as an input of k-means clustering algorithm.

In damage detection approaches, a damage index is associated with a decision-making (threshold) to ascertain with confidence the occurrence of a damage (Sohn et al. 2005). Unfortunately, environmental and operational variability render many of the proposed damage detection methods difficult to successfully be applied to aeronautical in-service structures. Indeed, one critical issue in SHM is to be able to differentiate the effects of variability inherent in the system and its environment from a potential damage. The threshold is then crucial to separate between the healthy and damaged states and also to minimize false-positive alarms. The outlier analysis (Worden et al. 2000), the extreme value statistics (Sohn et al. 2005), the T2 and Qstatistics (Mujica et al. 2011) are some of the methodologies used by the SHM community to establish such a threshold.

In this work, an original methodology of robust damage decision-making is proposed. It is based on: (1) ICA method to extract a sensitive feature in matrix form, (2) angle between ICA range subspaces to define a damage index, (3) matrix perturbation theory (MPT) to drive an incremental threshold. This proposed threshold unlike most other methods is not established using statistical approaches, but it is an analytical one. Mathematically speaking, let $\mathbf{A}$ be a sensitive matrix, subject to a perturbation that had let it to another one noted $\widetilde{\mathbf{A}}$. Through a perturbation model that relates matrix $\widetilde{\mathbf{A}}$ to $\mathbf{A}$, MPT gives an analytical upper bound to the deviation occurred in matrix functions A, i.e. eigenspaces/singular subspaces, eigenvalues/singular values (Stewart and Sun 1990).

The idea of addressing MPT for SHM decision-making has been recently introduced by the authors to define an analytical threshold associated to a principal component analysis (PCA) damage index (Hajrya and Mechbal 2013). In the present contribution, the PCA limits pointed up in their work have been overtaken by defining an ICA damage index. Unlike the PCA which 
considers only second-order statistics to obtain uncorrelated sources, ICA exploits the higher-order statistics embedded in the measurements to extract independent sources.

The present work falls within the SMSE project (Smart Materials and Structures for Electromagnetics), (SMSE 2010), where the objective is to evaluate the concept of new materials that enable the realization of compact and reconfigurable antenna composite structures. This adaptability assumes that these structures are equipped with sensors/actuators able to perform SHM, as well as active control (shape control and vibration rejection) tasks (Preumont 2002). This requirement leads us to use the Lead Zirconate Titanate piezoelectric ceramics (noted in what follow PZT) as actuators and sensors. Indeed, PZTs have good broadband sensing/actuation properties that make them extensively used for a wide range of frequency, including ultrasonic applications. It is also to be noticed that the SHM proposed approach is not limited to any frequency range, but within the project, the dynamic response of the monitored structures is generated at low frequency range to avoid interference with communication systems.

The proposed damage detection is an iterative one, and it relies on instantaneous knowledge of the structure. Before performing a health monitoring system, the structure is assumed to be in a healthy state. Typically, baseline measurements are recorded when the structure is pristine, and they are stored for comparison to future tested data for damage detection. When no longer damage is detected, the tested data become the new baseline database. The proposed approach is first presented through finite element (FE) simulations, where a composite plate bonded with PZTs is considered, and environmental variability's (temperature and noise changes) are introduced. Then, the method is applied to monitor two test benches: composite plates and a CLAS, subject respectively to impact damage and a delamination of the antenna array. For this last structure, interaction between the PZTs and antenna array has also been investigated in an anechoic chamber.

The layout of this paper is as follows: a description of the technique used to extract the feature using ICA is provided in section 2. In Section 3, the damage index is established. In Section 4, MPT is addressed to drive the SHM decision-making. The damage detection methodology is applied on a finite element model of an active composite plate, and it is presented in section 5 . Section 6 explores the proposed approach on the two test benches. A discussion regarding the proposed damage detection methodology is presented in section 7. Concluding remarks and future perspectives are drawn in Section 8.

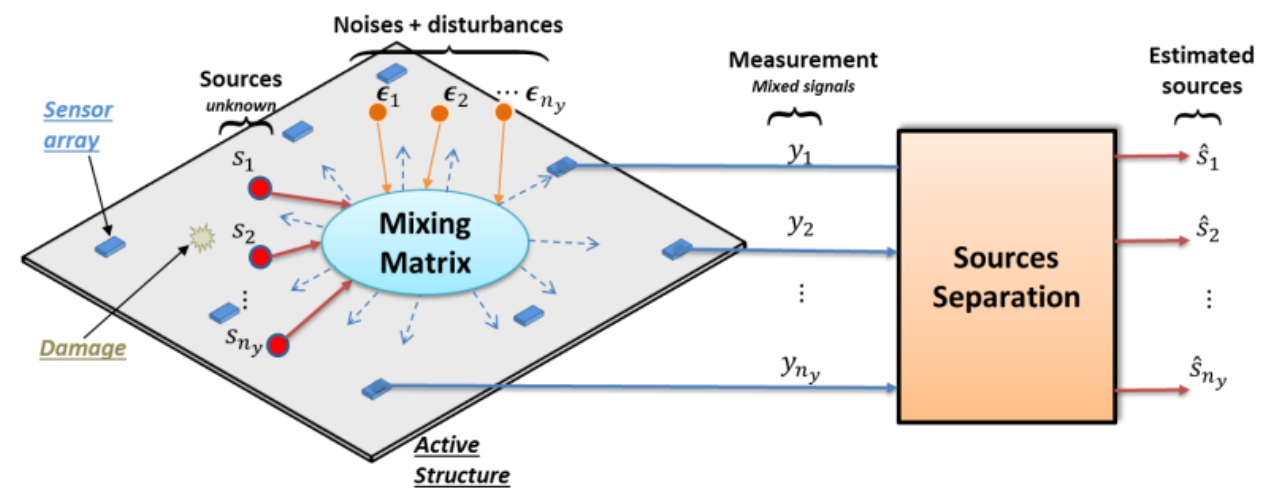

Fig. 1 BSS applied to damage structural monitoring 


\section{ICA for damage feature extraction}

\subsection{Brief overview of ICA theory}

The BSS model considered in this paper is a linear simultaneous mixture formulated as (Comon and Jutten 2010):

$$
\underline{\boldsymbol{y}}(k)=\mathbf{T} \underline{\boldsymbol{s}}(k)+\underline{\boldsymbol{\epsilon}}(k)
$$

where $\underline{\boldsymbol{y}}(k)=\left[\begin{array}{lll}y_{1}(k) & \ldots & y_{n_{y}}(k)\end{array}\right]^{T}$ is a zero mean measurement vector from $n_{y}$ sensors at time index $\bar{k}, \mathbf{T}$ is the mixing matrix, $\underline{\boldsymbol{s}}(k)=\left[\begin{array}{lll}s_{1}(k) & \ldots & s_{n_{y}}(k)\end{array}\right]^{T}$ is the sources vector and $\underline{\boldsymbol{\epsilon}}(k)=$ $\left[\epsilon_{1}(k) \ldots \epsilon_{n_{y}}(k)\right]^{T}$ represents all the uncertainties and disturbances effects.

In the present study, the unnoisy model is conducted. Uncertainties and perturbation's effects will be incorporated through the proposed threshold:

$$
\underline{\boldsymbol{y}}(k)=\mathbf{T} \underline{\boldsymbol{s}}(k)
$$

BSS is an estimation problem, that is accomplished by finding only from the observed data $\boldsymbol{y}(k)$ an estimated sources vector and a separating matrix noted respectively $\underline{\boldsymbol{r}}(k) \in \mathbb{R}^{n_{y} \times 1}$ and $\overline{\mathbf{W}} \in \mathbb{R}^{n_{y} \times n_{y}}$ :

$$
\underline{\boldsymbol{r}}(k)=\mathbf{W} \underline{\boldsymbol{y}}(k)
$$

In this paper, the sources are assumed to be temporally identically and independently distributed and non-Gaussian, which leads to the ICA method.

One way to solve the separation problem using ICA is to use the mutual information. Indeed, it is a measure of independence between variables of a random vector. It is always non-negative and zero, if and only if, the variables are statistically independent (Cover and Thomas 2006). Using the concept of negentropy introduced by Donoho (1981), the mutual information (noted $I(\underline{\boldsymbol{r}})$ ) between the components $r_{i}$ of a random vector $\underline{\boldsymbol{r}}$ is given by:

$$
I(\underline{\boldsymbol{r}})=I\left(\underline{\boldsymbol{r}}_{g}\right)+J(\underline{\boldsymbol{r}})-\sum_{i=1}^{n_{y}} J\left(r_{i}\right)
$$

where $I\left(\underline{\boldsymbol{r}}_{g}\right), J(\underline{\boldsymbol{r}}), J\left(r_{\mathrm{i}}\right), H(\underline{\boldsymbol{r}})$ and $H\left(r_{i}\right)$ represent respectively the mutual information of a Gaussian random vector, the joint negentropy, the marginal negentropy, the joint differential entropy and the marginal differential entropy. These quantities are defined by the following relations (Cover and Thomas 2006):

$$
\begin{gathered}
I\left(\underline{\boldsymbol{r}}_{g}\right)=\frac{1}{2} \ln \frac{\prod_{i=1}^{n_{y}} \sigma_{r_{g i}}^{2}}{\operatorname{det}\left(\boldsymbol{\Sigma}_{\boldsymbol{r}_{g}}\right)} \\
J(\underline{\boldsymbol{r}})=H\left(\underline{\boldsymbol{r}}_{\boldsymbol{g}}\right)-H(\underline{\boldsymbol{r}}), J\left(r_{i}\right)=H\left(r_{g i}\right)-H\left(r_{i}\right) \\
H(\underline{\boldsymbol{r}})=-\int_{\mathbb{R}^{n_{y}}} p_{\underline{\boldsymbol{r}}}(\underline{\xi}) \ln p_{\underline{\boldsymbol{r}}}(\underline{\xi}) d \underline{\xi}, \\
H\left(\underline{\boldsymbol{r}}_{g}\right)=\frac{1}{2} \ln \left\{(2 \pi e)^{\left.n_{y} \operatorname{det}\left(\boldsymbol{\Sigma}_{\underline{\boldsymbol{r}}_{g}}\right)\right\}}\right.
\end{gathered}
$$




$$
\begin{aligned}
& H\left(r_{i}\right)=-\int_{\mathbb{R}} p_{r_{i}}\left(\xi_{i}\right) \ln p_{r_{i}}\left(\xi_{i}\right) d \xi_{i}, \\
& H\left(r_{g i}\right)=\frac{1}{2} \ln \left\{2 \pi e \sigma_{r_{g i}}^{2}\right\}
\end{aligned}
$$

$p_{\underline{\underline{r}}}, p_{r_{i}}$ are respectively the joint and marginal probability density functions, $\boldsymbol{\Sigma}_{\underline{\boldsymbol{r}}_{g}}$ and $\sigma_{r_{g i}}^{2}$ are respectively the covariance matrix of the Gaussian random vector $\underline{\boldsymbol{r}}_{g}$ and the variance of its components $r_{g i}$.

The BSS problem comes down to estimate a source's vector $\underline{\boldsymbol{r}}=\left[r_{1} \cdots r_{n_{y}}\right]^{T}$ which follow a non-Gaussian distribution and whose components are statistically independent. The first step is the pre-whitening, which is the process of removing the correlation between the components of a data vector. It is accomplished by applying a linear transformation to the measured data vector $\underline{y}$ to produce a vector whose elements are mutually uncorrelated, and all have unit variance (in this case $\left.I\left(\underline{\boldsymbol{r}}_{g}\right)=0\right)$. Then, the separating matrix $\mathbf{W}$ is obtained by minimizing $I(\underline{\boldsymbol{r}})$ :

$$
\min _{\mathbf{W}} I(\underline{\boldsymbol{r}})=\min _{\mathbf{W}}\left\{J(\underline{\boldsymbol{r}})-\sum_{i=1}^{n_{y}} J\left(r_{i}\right)\right\}
$$

Based on approximate form of the negentropy quantities $J(\underline{\boldsymbol{r}})$ and $J\left(r_{i}\right)$, Gävert et al. (2005) have developed an efficient fixed-point toolbox for ICA (named FastICA). This algorithm calculates the separating matrix noted in what follow $\mathbf{W}_{\mathrm{ICA}}$, and allows then identification of the independent component vector (sources vector).

\subsection{Vibration analysis using ICA}

The dynamic response of a linear mechanical system with $n_{D O F}$ degrees of freedom is described by the flowing equation:

$$
\mathbf{M} \ddot{\ddot{x}}(t)+\mathbf{C} \underline{\dot{x}}(t)+\mathbf{K} \underline{\boldsymbol{x}}(t)=\underline{\boldsymbol{f}}(t)
$$

where $\mathbf{M}, \mathbf{C}$ and $\mathbf{K} \in \mathbb{R}^{n_{D O F} \times n_{D O F}}$ are respectively the mass, damping and stiffness matrices, $\underline{\boldsymbol{x}}(t) \in \mathbb{R}^{n_{D O F} \times 1}$ and $\boldsymbol{f}(t) \in \mathbb{R}^{n_{D O F} \times 1}$ are the time varying displacement response and the applied force.

The free vibration response $(\underline{\boldsymbol{f}}=\underline{\mathbf{0}})$ of a lightly damped structure can be described through the modal expansion form:

$$
\underline{\boldsymbol{x}}(t)=\sum_{i=1}^{n_{D O F}} \underline{\boldsymbol{\Psi}}_{i} a_{i} \exp \left(\xi_{i}\right) \cos \left(\omega_{i} t+\varphi_{i}\right)
$$

$\xi_{i}, \omega_{i}, \varphi_{i}$ represent respectively the damping ratio, natural frequency and phase angle of the mode shape $\underline{\boldsymbol{\Psi}}_{i}, a_{i}$ is a constant.

In discrete-time and matrix form, Eq. (11) is rewritten as:

$$
\underline{\boldsymbol{x}}(k)=\underline{\boldsymbol{\Psi}}(k)
$$

where $k$ is discrete-time index, $\boldsymbol{\Psi} \in \mathbb{R}^{n_{D O F} \times n_{D O F}}$ is the mode shape matrix, $\underline{\boldsymbol{q}}(k) \in \mathbb{R}^{n_{D O F} \times 1}$ is a vector containing the modal coordinates. 
From the previous relation, the correspondence between the modal expansion (Eq. (11)) and the static mixture BSS model (Eq. (2)) is straightforward: the modal coordinates act as virtual sources, and the mixing matrix reflects the mode shape matrix (Zhou and Chelidze 2007). BSS techniques can be seen as a non-parametric output-only modal identification method. Furthermore, as damages produce changes on the modal coordinates and the mode shape matrix; the virtual sources, the mixing and the separating matrices are also affected. Thereby, ICA can be used as a basis to build damage indices. In the sequel, a specific damage index based on monitoring the range subspaces of the separating matrix is proposed.

\section{Damage index}

Let $\mathbf{W}_{\text {ICA }}^{h}, \mathbf{W}_{\text {ICA }}^{u} \in \mathbb{R}^{n_{y} \times n_{y}}$ be the separating matrices, obtained respectively from data sensors of the structure in healthy and unknown state. For ease of reading, we abridge the notations:

$$
\mathbf{A}=\mathbf{W}_{\text {ICA }}^{h}, \overline{\mathbf{A}}=\mathbf{W}_{\text {ICA }}^{u}
$$

Now, using the singular value decomposition, the matrix $\mathbf{A}$ is rewritten as follows:

where:

$$
A=U \Gamma V=\left[\begin{array}{ll}
U_{1} & U_{2}
\end{array}\right]\left[\begin{array}{cc}
\Gamma_{1} & 0 \\
0 & \Gamma_{2}
\end{array}\right]\left[\begin{array}{ll}
V_{1} & V_{2}
\end{array}\right]^{T}=A_{1}+A_{2}
$$

$\mathbf{U}_{1}=\left[\underline{\boldsymbol{u}}_{11} \cdots \underline{\boldsymbol{u}}_{1 n_{r}}\right] \in \mathbb{R}^{n_{y} \times n_{r}}, \quad \boldsymbol{\Gamma}_{1}=\operatorname{diag}\left(\sigma_{1} \cdots \sigma_{n_{r}}\right), \quad \mathbf{V}_{1}=\left[\underline{\boldsymbol{v}}_{11} \cdots \underline{\boldsymbol{v}}_{1 n_{r}}\right] \in \mathbb{R}^{n_{y} \times n_{r}} \quad$ are respectively the matrix of left singular vectors, the matrix of singular values, the matrix of right singular vectors, associated to the principal subspaces (left and right) of the matrix A. These matrices are related to the main singular values, for example, concentrating more than $98 \%$ of the system total energy.

$\mathbf{U}_{2}=\left[\underline{\boldsymbol{u}}_{2\left(n_{r}+1\right)} \cdots \underline{\boldsymbol{u}}_{2 n_{y}}\right] \in \mathbb{R}^{n_{y} \times\left(n_{y}-n_{r}\right)}, \quad \boldsymbol{\Gamma}_{2}=\operatorname{diag}\left(\sigma_{n_{r}+1} \cdots \sigma_{n_{y}}\right)$, and

$\mathbf{V}_{2}=\left[\underline{\boldsymbol{v}}_{2\left(n_{r}+1\right)} \cdots \underline{\boldsymbol{v}}_{2 n_{y}}\right] \in \mathbb{R}^{n_{y} \times\left(n_{y}-n_{r}\right)}$ are respectively the matrix of left singular vectors, the matrix of singular values, and the matrix of right singular vectors, associated to the residual subspaces of matrix $\mathbf{A}$.

In the same way, the matrix $\overline{\mathbf{A}}$ is defined as:

$$
\overline{\mathbf{A}}=\overline{\mathbf{U} \boldsymbol{\Gamma V}}=\left[\begin{array}{ll}
\overline{\mathbf{U}}_{1} & \overline{\mathbf{U}}_{2}
\end{array}\right]\left[\begin{array}{cc}
\overline{\boldsymbol{\Gamma}}_{1} & \mathbf{0} \\
\mathbf{0} & \overline{\boldsymbol{\Gamma}}_{2}
\end{array}\right]\left[\begin{array}{ll}
\overline{\mathbf{V}}_{1} & \overline{\mathbf{V}}_{2}
\end{array}\right]^{T}=\overline{\mathbf{A}}_{1}+\overline{\mathbf{A}}_{2}
$$

As it was highlighted previously, the presence of damage causes a change in the mode shape matrix, and consequently a change in the separating matrix. Accordingly, the left and right principal subspaces of $\overline{\mathbf{A}}$ are deflected to those of $\mathbf{A}$ (Fig. 2). The proposed damage index (DI) is based on the calculus of the angle between the range subspaces of the matrix $\mathbf{A}_{1}$ and those of $\overline{\mathbf{A}}_{1}$. Let:

$R\left\{\overline{\mathbf{A}}_{1}\right\}, R\left\{\overline{\mathbf{A}}_{1}{ }^{T}\right\}$ be the ranges associated respectively to the left and right principal subspaces of matrix $\overline{\mathbf{A}}$,

$R\left\{\mathbf{A}_{1}\right\}, R\left\{\mathbf{A}_{1}{ }^{T}\right\}$ be the ranges associated respectively to the left and right principal subspaces of matrix $\overline{\mathbf{A}}$,

$\mathbf{P}_{R\left\{\overline{\mathbf{A}}_{1}\right\}}, \mathbf{P}_{R\left\{\overline{\mathbf{A}}_{1}{ }^{T}\right\}}, \mathbf{P}_{R\left\{\mathbf{A}_{1}\right\}}$ and $\mathbf{P}_{R\left\{\mathbf{A}_{1}{ }^{T}\right\}}$ be the orthogonal projection on theses ranges, defined as: 


$$
\begin{aligned}
& \mathbf{P}_{R\left\{\overline{\mathbf{A}}_{1}\right\}}=\overline{\mathbf{U}}_{1} \overline{\mathbf{U}}_{1}{ }^{T}, \mathbf{P}_{R\left\{\overline{\mathbf{A}}_{1}{ }^{T}\right\}}=\overline{\mathbf{V}}_{1} \overline{\mathbf{V}}_{1}{ }^{T} \\
& \mathbf{P}_{R\left\{\mathbf{A}_{1}\right\}}=\mathbf{U}_{1} \mathbf{U}_{1}{ }^{T}, \mathbf{P}_{R\left\{\mathbf{A}_{1}{ }^{T}\right\}}=\mathbf{V}_{1} \mathbf{V}_{1}{ }^{T}
\end{aligned}
$$

By introducing the following quantities:

$\left\|\sin \underline{\boldsymbol{\theta}}\left[R\left\{\overline{\mathbf{A}}_{1}\right\}, R\left\{\mathbf{A}_{1}\right\}\right]\right\|_{\mathrm{UI}}$ : the sinus angle between the range $R\left\{\mathbf{A}_{1}\right\}$ and $R\left\{\mathbf{A}_{1}\right\}$, $\left\|\sin \underline{\varphi}\left[R\left\{\overline{\mathbf{A}}_{1}{ }^{T}\right\}, R\left\{\mathbf{A}_{1}^{T}\right\}\right]\right\|_{\mathrm{UI}}$ : the sinus angle between the range $R\left\{\overline{\mathbf{A}}_{1}{ }^{T}\right\}$ and $R\left\{\mathbf{A}_{1}^{T}\right\}$.

where $\|.\|_{U I}$ denotes a general unitarily invariant norm, and the Euclidean norm $\|\cdot\|_{2}$ is used for the calculus.

The sinus angle norm of the aforementioned ranges is defined as (Golub and Van Loan 1983):

$$
\begin{gathered}
\mathrm{DI}_{\mathrm{ICA}}^{1}=\left\|\sin \underline{\boldsymbol{\theta}}\left[R\left\{\overline{\mathbf{A}}_{1}\right\}, R\left\{\mathbf{A}_{1}\right\}\right]\right\|=\left\|\left(\mathbf{I}_{n_{y}}-\mathbf{P}_{R\left\{\mathbf{A}_{1}\right\}}\right) \mathbf{P}_{R\left\{\overline{\mathbf{A}}_{1}\right\}}\right\| \\
\mathrm{DI}_{\mathrm{ICA}}^{2}=\left\|\sin \underline{\boldsymbol{\varphi}}\left[R\left\{\overline{\mathbf{A}}_{1}^{T}\right\}, R\left\{\mathbf{A}_{1}^{T}\right\}\right]\right\|_{2}=\left\|\left(\mathbf{I}_{n_{y}}-\mathbf{P}_{R\left\{\mathbf{A}_{1}^{T}\right\}}\right) \mathbf{P}_{R\left\{\overline{\mathbf{A}}_{1}^{T}\right\}}\right\|
\end{gathered}
$$

From these relations and considering the SHM problem of smart structures, a damage index is stated by the following proposition:

Proposal 1: Damage index

Consider a smart structure with $n_{y}$ sensors, damage could be detected by monitoring the following damage index:

$$
\mathrm{DI}_{\mathrm{ICA}}=\frac{\sqrt{\mathrm{DI}_{\mathrm{ICA}}^{1} \mathrm{DI}_{\mathrm{ICA}}^{2}}}{n_{r}}
$$

where $n_{r}$ is the number of principal components retained, and $\mathrm{DI}_{\mathrm{ICA}}^{1}, \mathrm{DI}_{\mathrm{ICA}}^{2}$ are defined in Eqs. (18) and (19).

Damage index based on the angle between subspaces was first proposed by De Boe and Golinval (2003). In their work, the authors have applied the QR decomposition ( $\mathbf{Y}=\mathbf{Q R})$ to the measurement matrix of the healthy and unknown states to get the cosines of the principal angles. The physical interpretation that underlies the proposed damage index is the fact that its definition is based on the ICA method, which is a procedure that allows to extract a basis for a modal decomposition (Zhou and Chelidze 2007). As modes are known to be sensitive to structural changes, it follows that the subspaces spanned by the ICA feature are deflected to those obtained from the healthy state. Furthermore, in the proposed damage index, we are monitoring the left and right subspaces of the separating matrix, i.e., $R\left\{\mathbf{A}_{1}\right\}$ and $R\left\{\mathbf{A}_{1}^{T}\right\}$, in the case where one of them is more sensitive to the presence of a damage.

\section{Analytical threshold}

Now that the damage index is defined, a threshold has to be established. Indeed, as stated previously, a critical issue in SHM is to be able to differentiate the disturbance effects that a healthy monitored structure undergoes from damage. Furthermore, environmental disturbances such as measurement noises, temperature variations could lead to false-positive alarms or missing detection. To overcome this drawback, probabilistic decision making approaches are in general 
used, which suppose a set of probabilistic models and assumptions. However, these models need in general large database to correctly approximate them.

In this work, since the proposed damage index deals mainly with matrix decomposition and subspace projections, we propose to bind analytically the deviation that appears in the subspaces of the separating matrix, when the structure undergoes a low level environmental variability. Thereby, MPT is addressed to define an analytical threshold and to get rid of statistical assumptions.

MPT considers how matrix functions such as subspaces change when the matrix is subject to perturbations (Stewart and Sun 1990):

$$
\widetilde{\mathbf{A}}=\mathbf{A}+\delta \mathbf{A}
$$

where the matrix $\delta \mathbf{A}$ describes the variation that matrix $\mathbf{A}$ is subjected due to disturbances. Then, the objective is to define a robust average that estimates how much the damage index $\mathrm{DI}_{\text {ICA }}$ (Eq. (20)) is affected.

To drive the proposed analytical bound, the early work of Wedin (1972) on perturbed matrices was opted. The idea is to estimate $\|\delta \mathbf{A}\|$ by performing several tests or simulations on the healthy structure and by evaluating the gap between specific singular values in order to define an upper bound for the damage index.

Proposal 2: Analytical threshold and detection rate

Assume that $\exists \eta \geq 0$, and $\delta>0$, an upper threshold noted $\beta_{\mathrm{ICA}}$ of the damage index is defined as:

$$
\beta_{\mathrm{ICA}}=\frac{\sqrt{\left(\varepsilon+\eta \widetilde{\mathrm{DI}}_{\mathrm{ICA}}^{2}\right)\left(\varepsilon+\eta \widetilde{\mathrm{DI}}_{\mathrm{ICA}}^{1}\right)}}{(\eta+\delta) n_{r}}
$$

where $\widetilde{\mathrm{DI}}_{\mathrm{ICA}}^{1}$ and $\widetilde{\mathrm{DI}}_{\mathrm{ICA}}^{2}$ are defined in the same way as in Eqs. (18)-(19) by replacing $\overline{\mathbf{A}}_{1}$ by $\widetilde{\mathrm{A}}_{1}$, $\varepsilon$ quantifies the magnitude of the environmental disturbances and it is defined in Eq.(31), $n_{r}$ is the number of principal components retained, and the two scalars $\eta$ and $\delta$ are defined as:

$$
\begin{gathered}
\eta \geq \sigma_{\max }\left(\mathbf{A}_{2}\right) \\
\eta+\delta \leq \sigma_{\min }\left(\widetilde{\mathbf{A}}_{1}\right)
\end{gathered}
$$

The DI defined in Eq. (20) and its associated threshold of Eq. (22) define the following detection rate:

$$
\mathrm{R}_{\mathrm{ICA}}=\frac{\mathrm{DI}_{\mathrm{ICA}}}{\beta_{\mathrm{ICA}}}
$$

if $\mathrm{R}_{\mathrm{ICA}}>1$ then the structure is damaged, otherwise it is healthy.

\section{Derivation:}

The proposed analytical threshold is derived following three major steps:

First step:

Consider the variation $\delta \mathbf{A}$ that the separating matrix $\mathbf{A}$ is subjected due to the environmental disturbances. To describe this variation, a second test/simulation is performed on the healthy state of the structure, ICA algorithm is then applied to determine the new separating matrix noted $\widetilde{\mathbf{A}}$. This matrix is rewritten as: 


$$
\widetilde{\mathbf{A}}=\widetilde{\mathbf{U}} \widetilde{\boldsymbol{\Gamma}} \widetilde{\mathbf{V}}=\left[\begin{array}{ll}
\widetilde{\mathbf{U}}_{1} & \widetilde{\mathbf{U}}_{2}
\end{array}\right]\left[\begin{array}{cc}
\widetilde{\boldsymbol{\Gamma}}_{1} & \mathbf{0} \\
\mathbf{0} & \tilde{\boldsymbol{\Gamma}}_{2}
\end{array}\right]\left[\begin{array}{ll}
\widetilde{\mathbf{V}}_{1} & \widetilde{\mathbf{V}}_{2}
\end{array}\right]^{T}=\widetilde{\mathbf{A}}_{1}+\widetilde{\mathbf{A}}_{2}
$$

Define the following residual matrices:

$$
\begin{aligned}
& \mathbf{R}_{11}=\mathbf{A} \widetilde{\mathbf{V}}_{1}-\widetilde{\mathbf{U}}_{1} \widetilde{\boldsymbol{\Gamma}}_{1}=-\delta \mathbf{A} \widetilde{\mathbf{V}}_{1} \\
& \mathbf{R}_{21}=(\mathbf{A})^{T} \widetilde{\mathbf{U}}_{1}-\widetilde{\mathbf{V}}_{1}\left(\widetilde{\boldsymbol{\Gamma}}_{1}\right)^{T}=-(\delta \mathbf{A})^{T} \widetilde{\mathbf{U}}_{1}
\end{aligned}
$$

and evaluate their norms, i.e.:

$$
\begin{aligned}
\left\|\mathbf{R}_{11}\right\| & =\left\|\delta \mathbf{A} \widetilde{\mathbf{V}}_{1}^{h}\right\| \\
\left\|\mathbf{R}_{21}\right\| & =\left\|(\delta \mathbf{A})^{T} \widetilde{\mathbf{U}}_{1}^{h}\right\|
\end{aligned}
$$

The scalar $\varepsilon$ is then obtained by:

$$
\varepsilon=\max \left(\left\|\mathbf{R}_{11}\right\|,\left\|\mathbf{R}_{21}\right\|\right)
$$

Second step:

Assume now, that there exist two scalars: $\eta \geq 0$ and $\delta>0$, such that the relations defined in Eqs. (23)-(24) hold, then using the results demonstrated in (Wedin 1972), the following relations are derived:

$$
\begin{aligned}
& \widetilde{\mathrm{DI}}_{\mathrm{ICA}}^{1}=\left\|\sin \underline{\boldsymbol{\theta}}\left[R\left\{\widetilde{\mathbf{A}}_{1}\right\}, R\left\{\mathbf{A}_{1}\right\}\right]\right\|_{2} \leq \frac{\varepsilon+\eta \widetilde{\mathrm{DI}}_{\mathrm{ICA}}^{2}}{\eta+\delta} \\
& \widetilde{\mathrm{DI}}_{\mathrm{ICA}}^{2}=\left\|\sin \underline{\boldsymbol{\varphi}}\left[R\left\{\widetilde{\mathbf{A}}_{1}^{T}\right\}, R\left\{\mathbf{A}_{1}^{T}\right\}\right]\right\|_{2} \leq \frac{\varepsilon+\eta \widetilde{\mathrm{DI}}_{\mathrm{ICA}}^{1}}{\eta+\delta}
\end{aligned}
$$

Third step:

The damage index defined in Eq. (20) is calculated from the separating matrix of the healthy and unknown states $\mathbf{A}, \overline{\mathbf{A}}$, while the term $\widetilde{\mathrm{DI}}_{\mathrm{ICA}}$ is calculated from the separating matrices $\mathbf{A}, \widetilde{\mathbf{A}}$ of the healthy state under disturbances. Consequently, the term $\widetilde{\mathrm{DI}}_{\text {ICA }}$ satisfies:

$$
\widetilde{\mathrm{DI}}_{\mathrm{ICA}} \leq \frac{\sqrt{\left(\varepsilon+\eta \widetilde{\mathrm{DI}}_{\mathrm{ICA}}^{2}\right)\left(\varepsilon+\eta \widetilde{\mathrm{DI}}_{\mathrm{ICA}}^{1}\right)}}{(\eta+\delta) n_{r}}
$$

The general framework of the proposed damage monitoring procedure is outlined in Fig. 3. It is to be noticed that the proposed approach is an unsupervised learning method, which implies that the data from a damaged state are not used to build this threshold. Moreover, it is an incremental and iterative procedure. In practice, the design procedure depicted in Fig. 3 is first performed, and then if there is no longer detected damage, the unknown state becomes the baseline, and the design is repeated. The incremental procedure, summarized in Fig. 4 permits to ensure that the healthy baseline structure is always updated. 


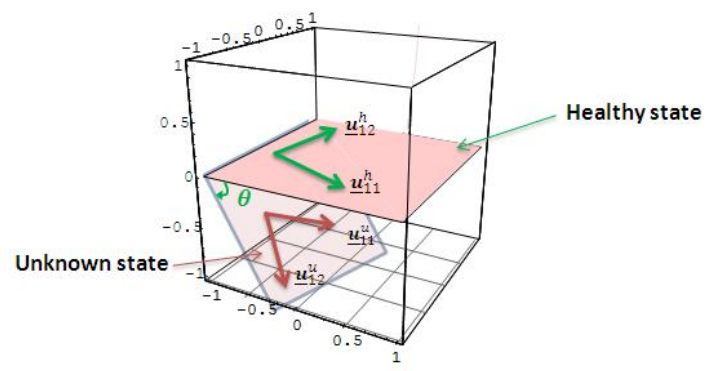

Fig. 2 Angle between subspaces

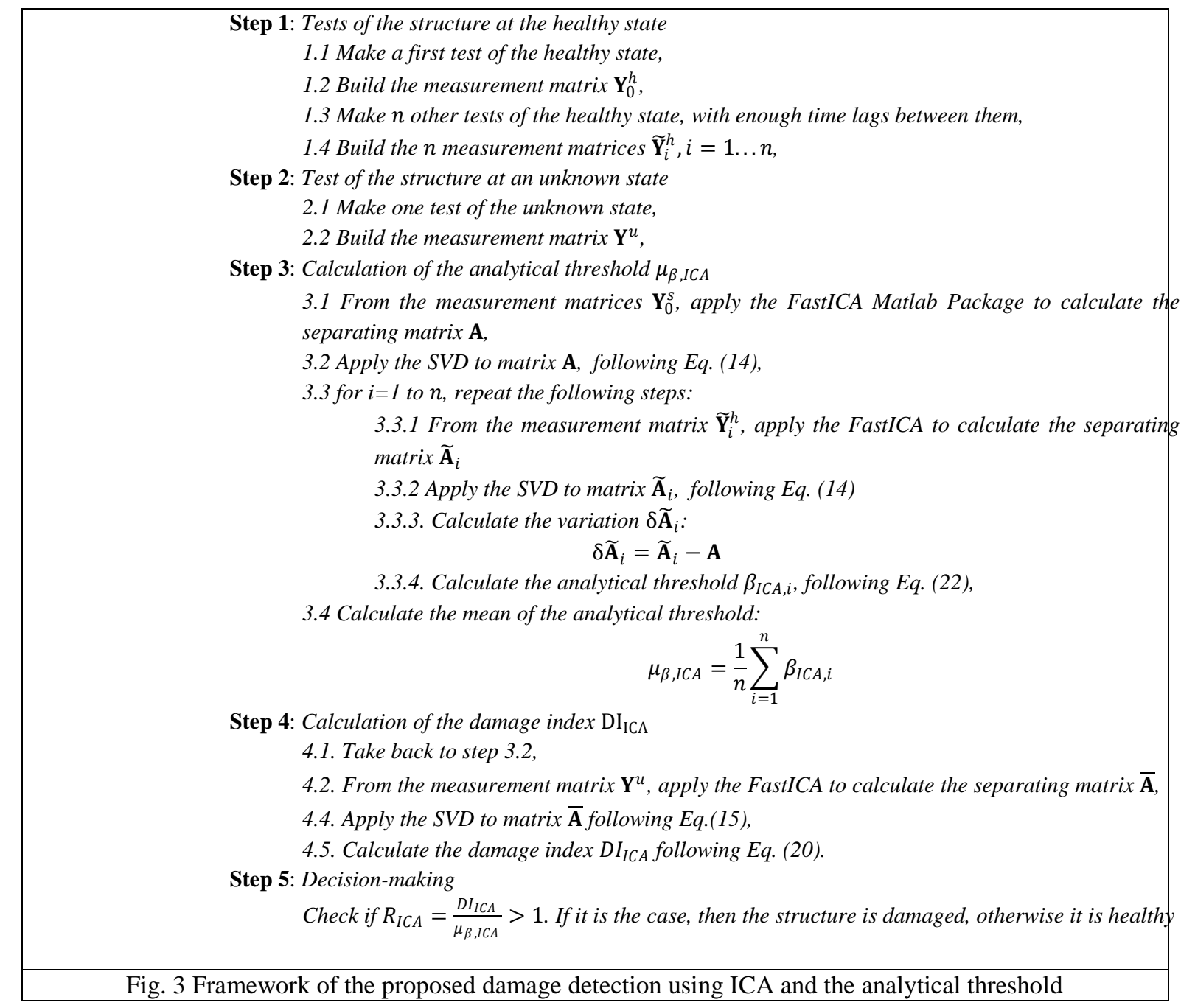




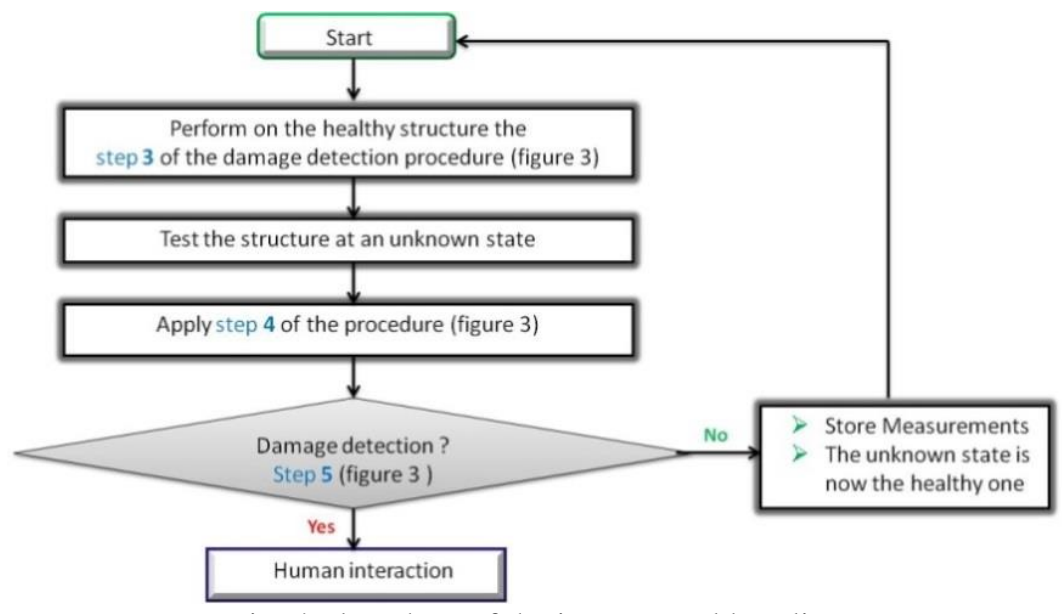

Fig. 4 Flowchart of the incremental baseline

\section{Simulation results on a composite plate}

\subsection{Composite plate specimen}

The test specimen is of a fuselage piece. It is a composite plate of dimension $400 \times 300 \times$ $2 \mathrm{~mm}^{3}$, and made up of 16 layers carbon epoxy material. The layer sequences are: $\left[0_{2}^{\circ} / 45_{2}^{\circ} /\right.$ $\left.-45_{2}^{\circ} / 90_{2}^{\circ} / 90_{2}^{\circ} /-45_{2}^{\circ} / 45_{2}^{\circ} / 0_{2}^{\circ}\right]$. The mechanical properties of the composite material are illustrated in Table 1. Table 2 depicts the mechanical and electrical properties of the PZT, type PZ29 (Ferroperm 2009). Using the controllability and observability Gramians, an optimal placement of the PZTs with dimension of $30 \times 20 \times 0.2 \mathrm{~mm}^{3}$ was proposed by the authors (Hajrya et al. 2010) (see Fig. 5(a)).

Table 2 Mechanical properties of the composite material

\begin{tabular}{|c|c|c|c|c|c|c|c|}
\hline Property & $E_{1}(\mathrm{GPa})$ & $\begin{array}{c}E_{2}=E_{3} \\
(\mathrm{GPa})\end{array}$ & $\begin{array}{c}G_{12}=G_{13} \\
(\mathrm{GPa})\end{array}$ & $G_{23}(\mathrm{GPa})$ & $v_{12}=v_{13}$ & $v_{23}$ & $\rho\left(\mathrm{Kg} / \mathrm{m}^{3}\right)$ \\
\hline Value & 127.7 & 7.217 & 5.712 & 2.614 & 0.318 & 0.38 & 1546 \\
\hline
\end{tabular}

Table 3 Mechanical and electrical properties of the piezoelectric patches PZ29 (Ferroperm 2009)

\begin{tabular}{|c|c|c|c|c|c|c|}
\hline Property & $\mathrm{E}(\mathrm{GPa})$ & $v$ & $\rho\left(\mathrm{Kg} / \mathrm{m}^{3}\right)$ & $\mathrm{d}_{31}(\mathrm{C} / \mathrm{N})$ & $\mathrm{d}_{33}(\mathrm{C} / \mathrm{N})$ & ${\text { Curie temperature } \mathrm{C}^{\circ}}^{-10}$ \\
\hline Value & 58.8 & 0.3 & 7460 & $-2.43 \times 10^{-10}$ & $5.74 \times 10^{-10}$ & 235 \\
\hline
\end{tabular}

\subsection{FE modeling of the active composite plate}

In order to outline the environment effects and to test the validity of the proposed damage detection algorithm, a detailed FE model of the active plate has been developed using the Structural Dynamics Toolbox (SDTools). The developed model is devoted to simulate vibratory data. 
SDTools is a FE toolbox developed in Matlab environment. It has a specific parameterized FE model of PZT elements with electromechanical coupling, that allows to perform predictive behavior of the active structure (Balmes and Deraemaeker 2013). For wave propagation, computation of periodic solutions using Fourier/Floquet solutions is proposed in the toolbox. Moreover, following the approach used by Valliappan et al. (1990), a parametrical damage is introduced. The damage is represented by a reduction of material properties in a chosen area (see Fig. 5(a)). To introduce this damage, we used a strategy inspired by the one employed in SDTools for PZT modeling. Indeed, a parameterized damaged patch is generated with a specific mesh, where its dimension and mechanical properties could then be changed and adjusted (Fig. 5(b)). The introduction of this patch before changing its properties does not change the modal properties of the structure in the frequency range of interest (Fig. 5(c)).

The FE model is under free-free boundary and consists of 195 elements; each element has dimension of $15 \times 10 \mathrm{~mm}^{2}$. This mesh size is compatible with the frequency range of interest ([0 $10 \mathrm{kHz}]$ ). The model was calculated with $n_{m}=50$ first modes with proportional damping, satisfying Caughey's criteria (Adhikari 2006).

In both of simulations and experiments of the composite plate, the signal excitation used is a Schroeder signal, with frequency range of [0 $2 \mathrm{kHz}$. This signal has a flat power spectral density over its frequency range (Fig. 6). The choice of this frequency range was imposed by the real time acquisition system used in the experiments described in the next section. The simulated actuation was applied through PZT 7, while the other PZTs are used as sensors. The characteristic of the simulated data are: sampling frequency $f_{s}=8 \mathrm{kHz}$, and $N=2^{14}$ time samples.

\subsection{Baseline using noise and temperature changes}

To calculate the novelty analytical threshold, 50 simulations were conducted by considering the healthy plate model under different values of noise and temperature. An inspection of the nature of the experimental noise was first exanimated in the test bench described in section 6. For this purpose, tests on the experimental healthy and damaged composite plates were conducted by recording signal sensors in the case where no excitation is acting on the structure. The idea here is to use the same noise that the one acting on the structure. Using the marginal negentropy defined in Eq. (6), the non-Gaussianity of the measured noises was quantified. The results are illustrated in Tables 3-4, and show that the negentropy of the measured noises are different from zero. These results conclude the fact that the experimental noise is non-Gaussian. Therefore, in the sequel, a non-Gaussian noise will be added to all the finite element simulations to mimic a type of disturbance.

With the inherent anisotropy of composite materials, any attempt to simulate the effect of environmental disturbance like temperature requires relevant experimental data from the structure in an enclosed heated space. These data will feed the FE model to yield reasonably accurate prediction. However, it is expected that the first-order thermal effects in an instrumented composite structure will be associated with the difference between the evolution of constitutive behaviour of the composite matrix, its fibers and the PZT. As a rough approximation, one can consider varying moduli for the composite plate with fixed mechanical properties for the PZT.

Following this modeling manner, the moduli $\left(E_{1}, E_{2}, G_{12}, G_{23}\right)$ were varied by a percentage of $\theta \in\left[\begin{array}{ll}0.04 & 1.44\end{array}\right] \%$, with a step of $0.0071 \%$ and for each of these simulations, different variance of random non-Gaussian noise were added (from 1\% to 25.5\%). Once the baseline built, step 3 of 
the framework presented in Fig.3 was applied, and the novelty analytical threshold was calculated: $\mu_{\beta, I C A}=0.0673$.

\subsection{Simulation results of damage scenarios and test of false-positive alarms}

In order to test the performances of the proposed method, 54 simulations with different damaged elements and environmental disturbances were conducted. The first 4 simulations (no ${ }^{\circ} 51-$ no $^{\circ} 54$ ) concern a reduced stiffness of $4 \%$ for damages $D 1, . . D 5$ (see Fig. 5(a)), and with different temperature change and non-Gaussian noise. The other simulations (no ${ }^{\circ} 55-$ no $\left.^{\circ} 104\right)$ were performed to test the damage detection methodology regarding false-positive alarms. These simulations were performed by varying the moduli $\left(\mathrm{E}_{1}, \mathrm{E}_{2}, \mathrm{G}_{12}, \mathrm{G}_{23}\right)$ by a percentage $\theta \in$ [0.08 1.48]\%, with a step of $0.0071 \%$. It is to be noted that the aforementioned simulations do not belong to those used to build the baseline test. Fig. 7 depicts some of the results associated to these simulations. On one hand, the damaged states are well detected and separated from the analytical threshold. On the other hand, no false-positive alarms were noted: the analytical threshold is robust to the simulated new perturbations.

Table 4 Negentropy of the measured noise for different sensors: healthy composite plate

\begin{tabular}{|l|c|c|c|c|c|c|c|c|}
\hline Sensor & 1 & 2 & 3 & 4 & 5 & 8 & 9 & 10 \\
\hline Negentropy & 1.7845 & 1.7849 & 1.7977 & 1.8632 & 2.1086 & 1.8464 & 2.0650 & 2.7648 \\
\hline
\end{tabular}

Table 4 Negentropy of the measured noise for different sensors: damaged composite plate

\begin{tabular}{|l|c|c|c|c|c|c|c|c|}
\hline Sensor & 1 & 2 & 3 & 4 & 5 & 8 & 9 & 10 \\
\hline Negentropy & 1.7835 & 1.7847 & 1.7883 & 1.8044 & 3.1618 & 1.7476 & 1.7904 & 1.7908 \\
\hline
\end{tabular}

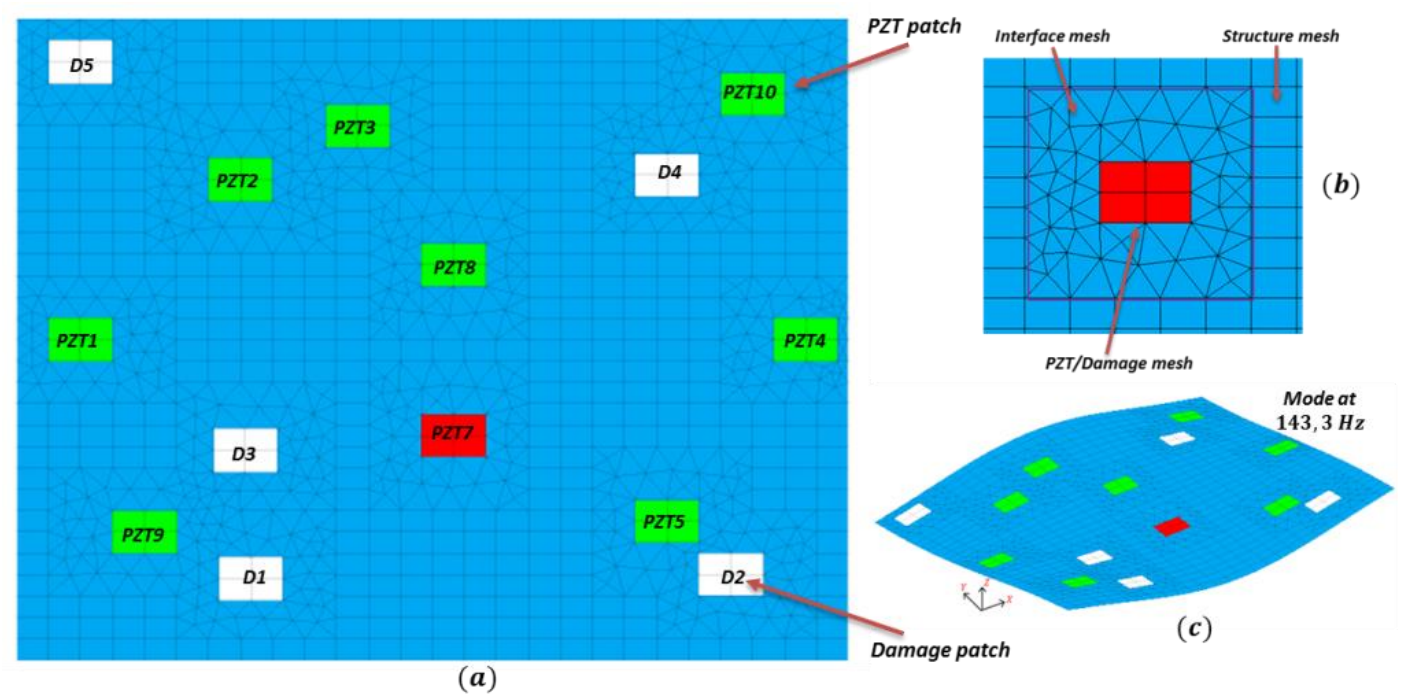

Fig. 5: (a) Finite element model of the composite plate bounded with PZT, (b) a zoom on the PZT element, (c) a simulated mode of the composite plate 


\section{Experimental applications}

\subsection{Active composite plates}

Following the optimal placement results (Hajrya et al. 2010), PZTs were glued on two composite plates: a healthy (Fig. 8 (a)) and a damaged one (Fig. 8 (b)). The two plates have the same characteristics and dimensions: $400 \times 300 \times 2 \mathrm{~mm}^{3}$, except that in one of them, damage was introduced. This later has undergone an impact damage (diameter of $5 \mathrm{~mm}$ ) produced by a calibrated impact device. In order to test the proposed approach regarding false-positive alarms, two additive masses were used for boundary condition changes: see Fig. 8 (c). These masses represent $0.5 \%$ and $0.2 \%$ of the total composite plate weight.

The input excitation and the data acquisition were performed using a voltage amplifier (TREK Model 601C) and charge amplifiers (type 5011B). The actuation and sensor records were done following the same procedure as in the simulation: actuation using the Schroeder signal, frequency range of $\left[\begin{array}{ll}0 & 2 \mathrm{kHz}\end{array}\right]$ (Fig. 6), sampling frequency $f_{s}=8 \mathrm{kHz}$, and $N=2^{14}$ time samples. To calculate the analytical threshold, $n=40$ tests with enough time lags between them were conducted on the healthy plate.

The vibratory data acquired from the sensors of the composite plates (see Figs. 9-10) were transformed into features through the ICA method. From many tests of the healthy and damaged plates, the damage index and the analytical threshold defined respectively in Eqs. (20) and (22) were calculated. Fig. 11 depicts the obtained results; one can see that the impact damaged tests are well detected and separated from the healthy sate. Concerning the two additive masses used for boundary conditions changes, the proposed approach is robust regarding them.

\subsection{Conformal load-bearing antenna structure (CLAS)}

The second test bench realized concerns a CLAS. The dimension of the host structure is $800 \times 150 \times 2 \mathrm{~mm}^{3}$, and it is made up from the same material and number of layers as the composite plates. In addition to the PZTs, an antenna network is bonded on it. Figs. 12(a)-(b) show respectively the antenna network of the CLAS and the seven PZT bonded on it.

Before applying the proposed damage detection methodology, a radiation pattern and a measure of bending and torsion strains using the PZT sensors were conducted with the partners of our project (SMSE 2010). These tests were done simultaneously in an anechoic chamber, and they were conducted in order to check if there is any coupling between the electromagnetic phenomena of the antenna network and the electrical information transmitted by the PZT sensors. First of all, a strain measurement of bending and torsion were conducted before and during the radiation pattern (Fig. 13). Then, a radiation pattern was done before and during the strain measurements (Fig. 14). The results obtained show that the two phenomena can coexist in the same time without any interaction.

Once this study performed, the damage detection procedure was applied. The healthy baseline state was first built. The input excitation consists in a signal pulse with $1 \mathrm{~ms}$ width, sampling frequency $f_{s}=100 \mathrm{kHz}$ and $N=2^{16}$ time samples were recorded. Once the healthy baseline was set, damage was introduced. In this case, a delamination between the antenna part and the host structure was provoked using a buckling device (see Fig. 15(a)-(b)). It is to be noted that the baseline was built through 14 tests with enough time lag between them. Fig. 16 depicts the damage 
detection results of the CLAS: one can see that the delamination of the antenna array is well detected and distinguished from the healthy tests done.
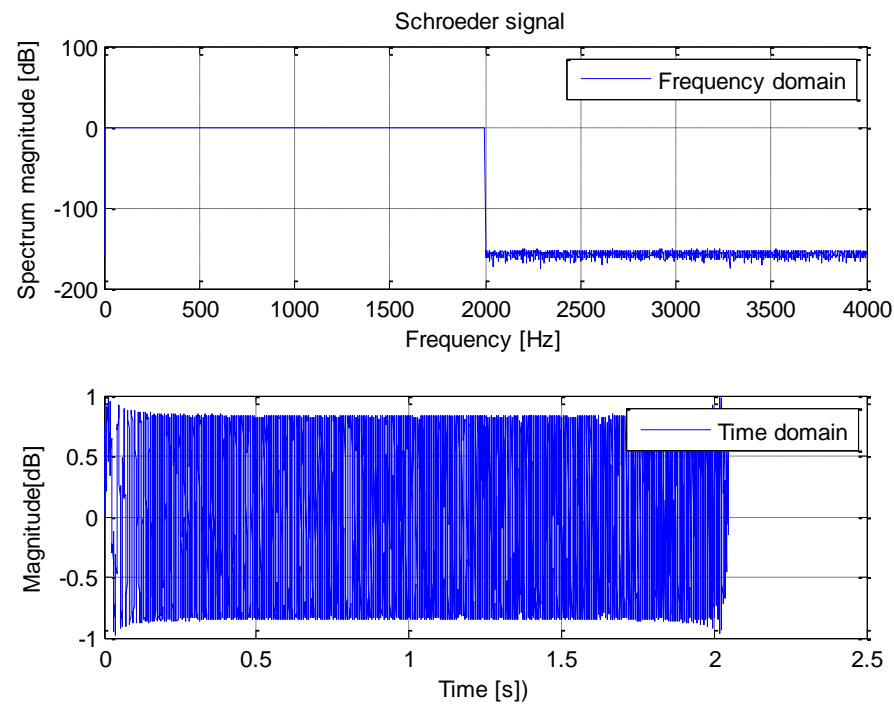

Fig. 6 Time and frequency characteristics of the input excitation: Signal used for FE simulations and composite plates experiments

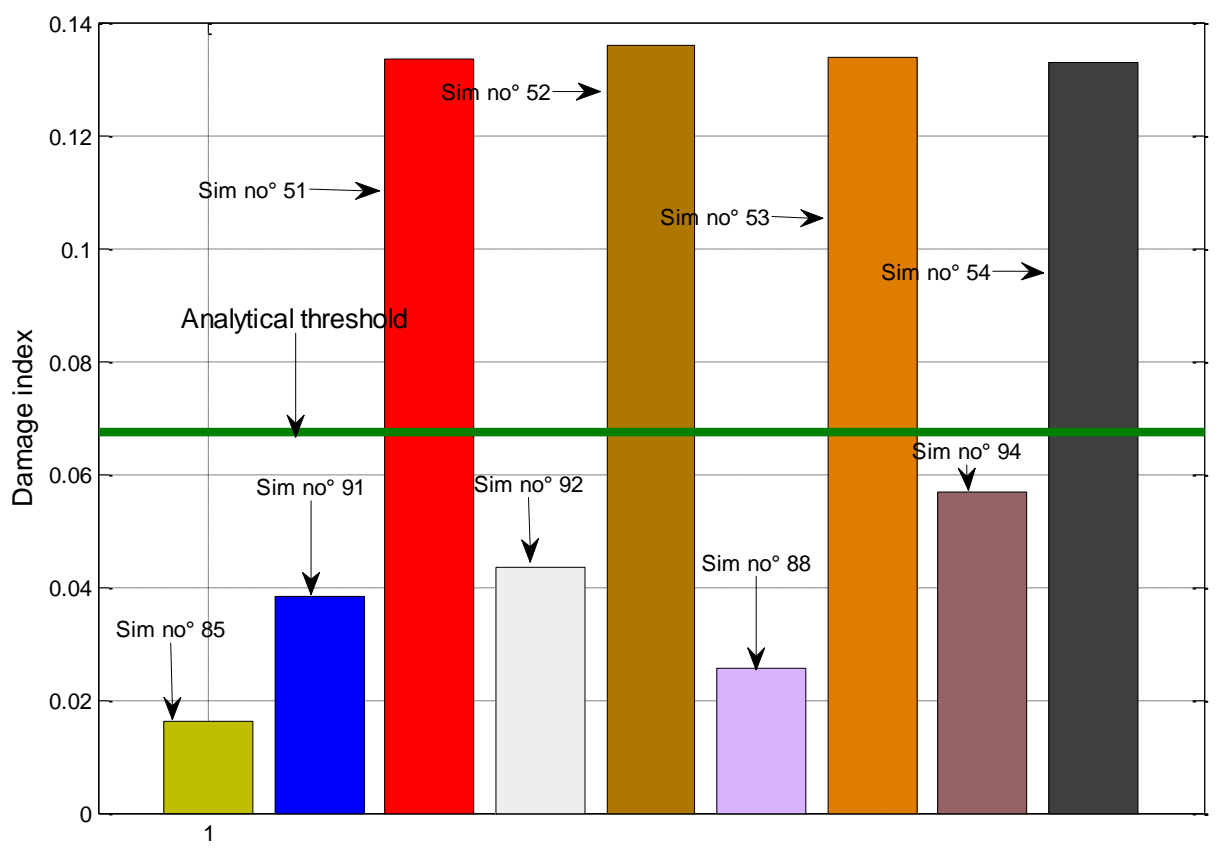

Fig. 7 Results of simulated damage scenario 

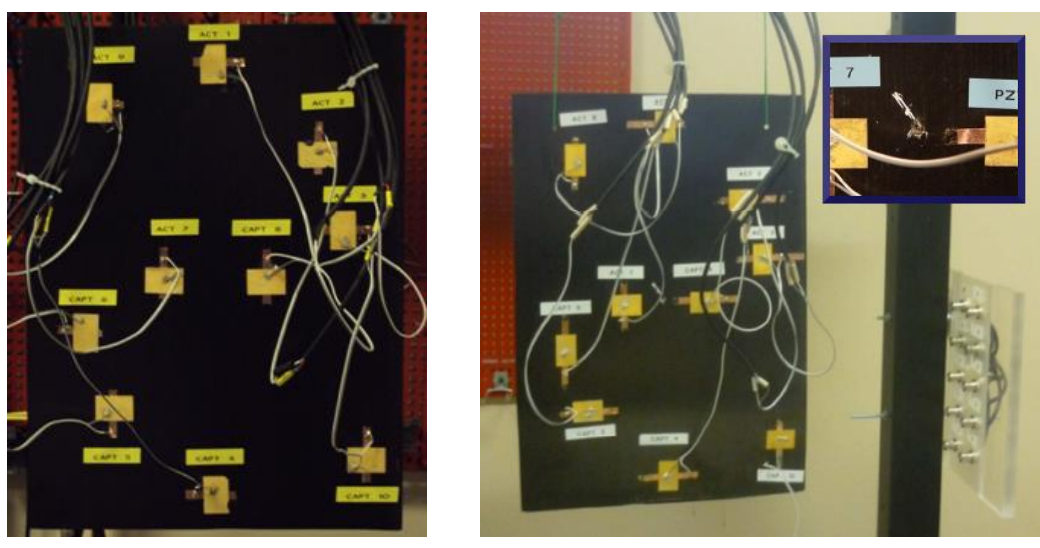

(a) Healthy

Fig. 8 Composite plates bounded with PZTs
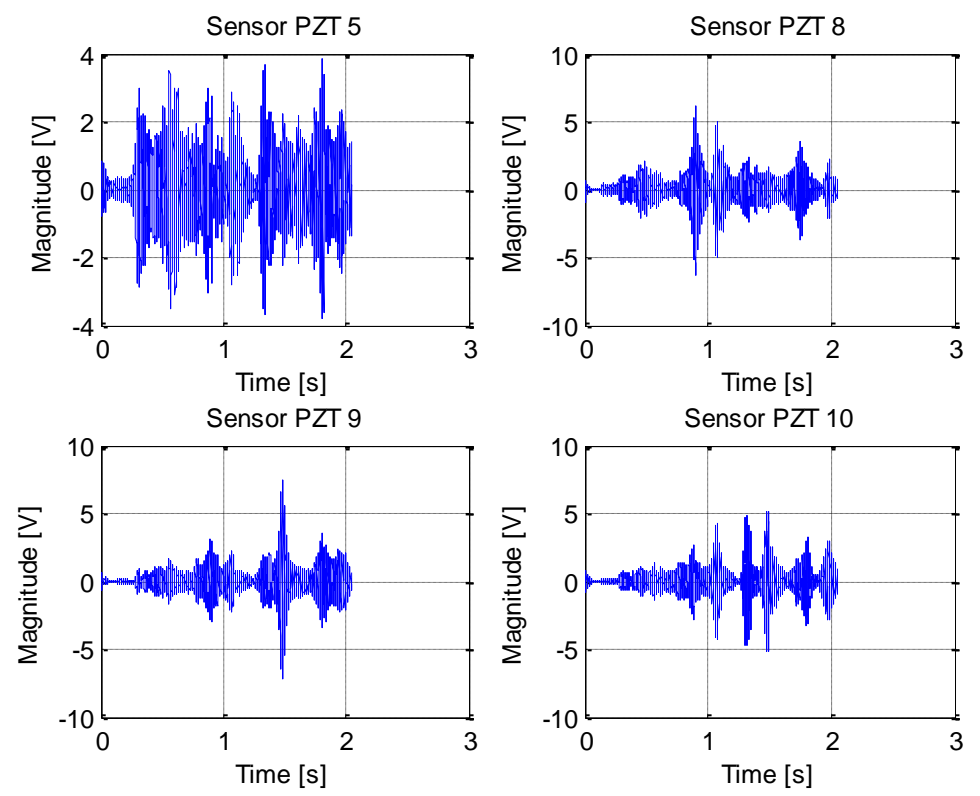

Fig. 9 Dynamic response acquired from some sensors of the healthy plate 

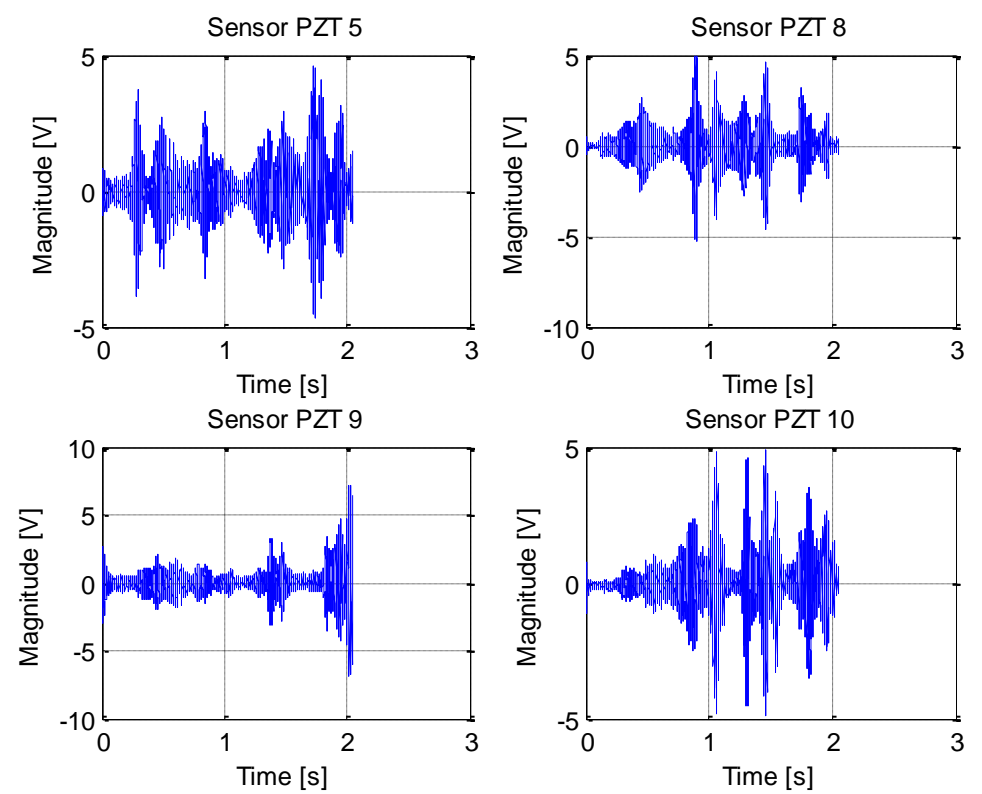

Fig. 10 Dynamic response acquired from some sensors of the damaged plate

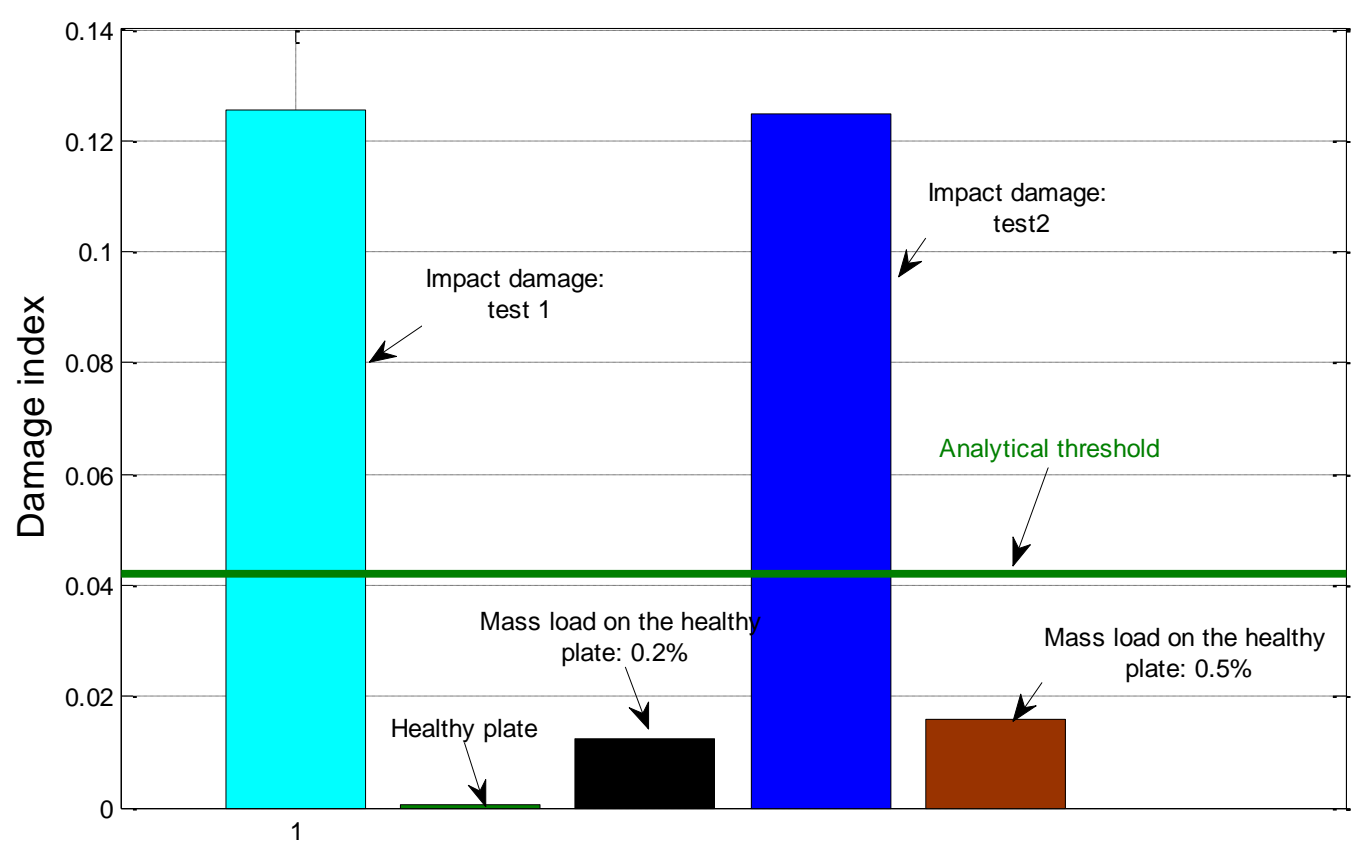

Fig. 11 Results of the damage detection of the composite plates 


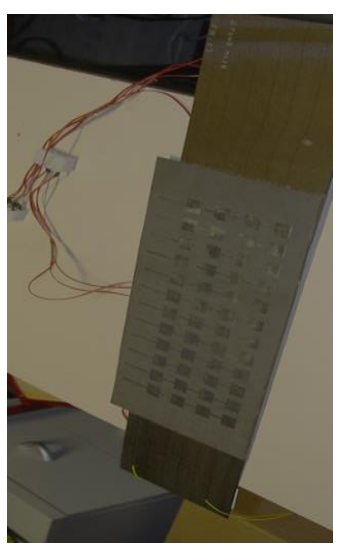

(a) Antenna network of the CLAS

Fig. 12 Conformal load-bearing antenna structure

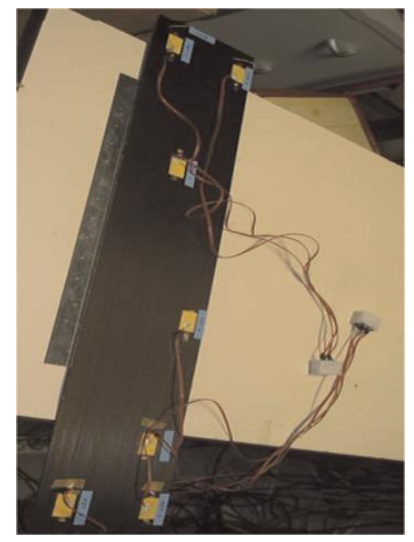

(b) CLAS bounded with PZTs

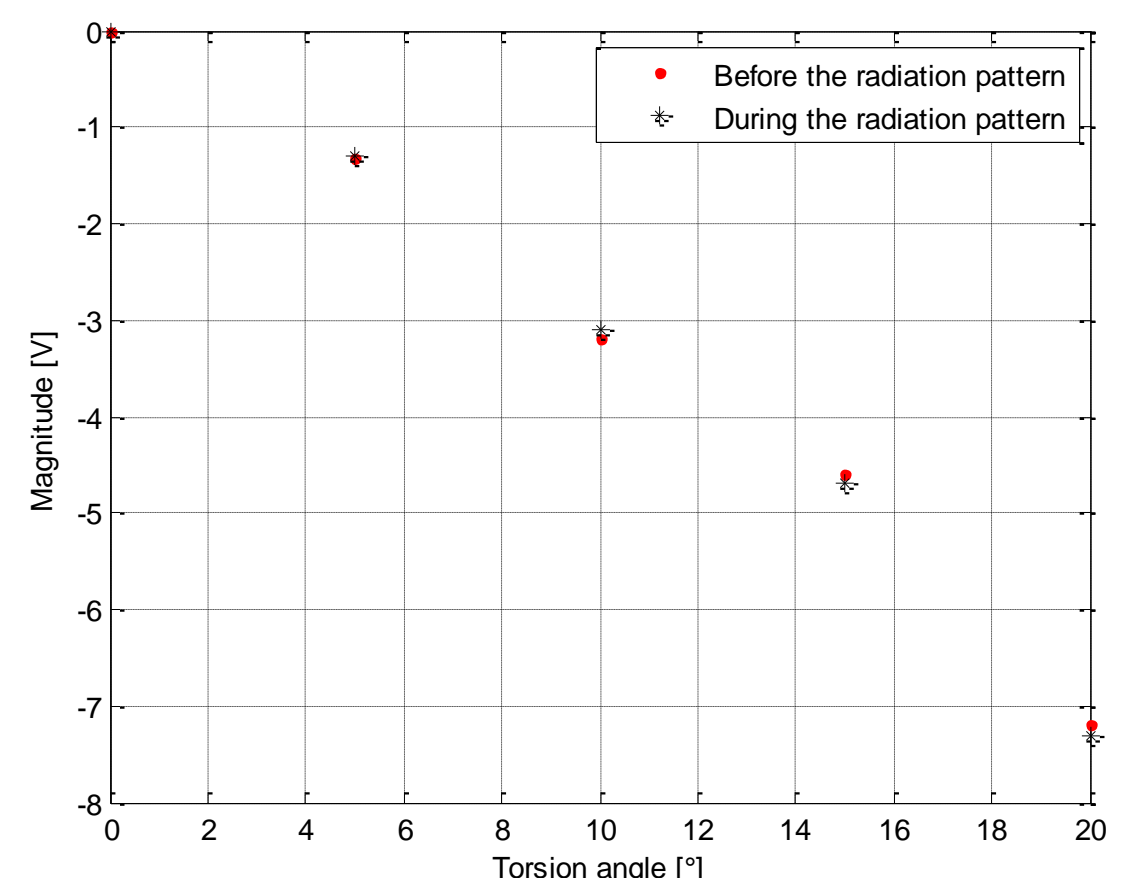

Fig. 13 Response of PZT sensor due to a torsional strain 


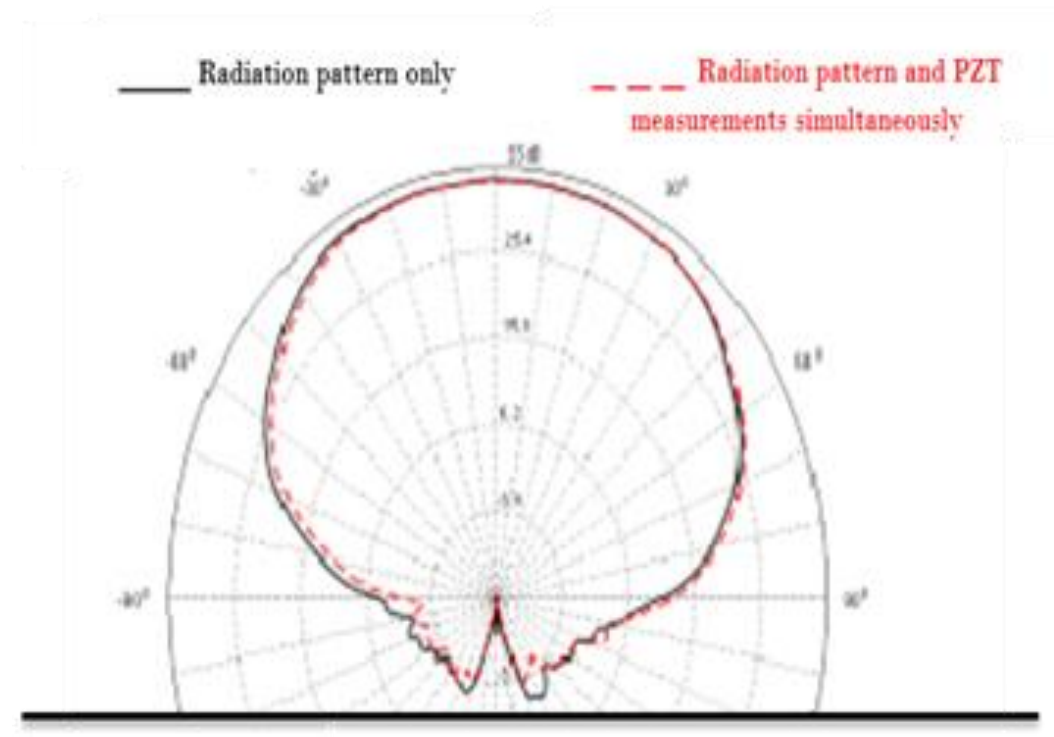

Fig. 14 Radiation pattern measurements (SMSE 2010)
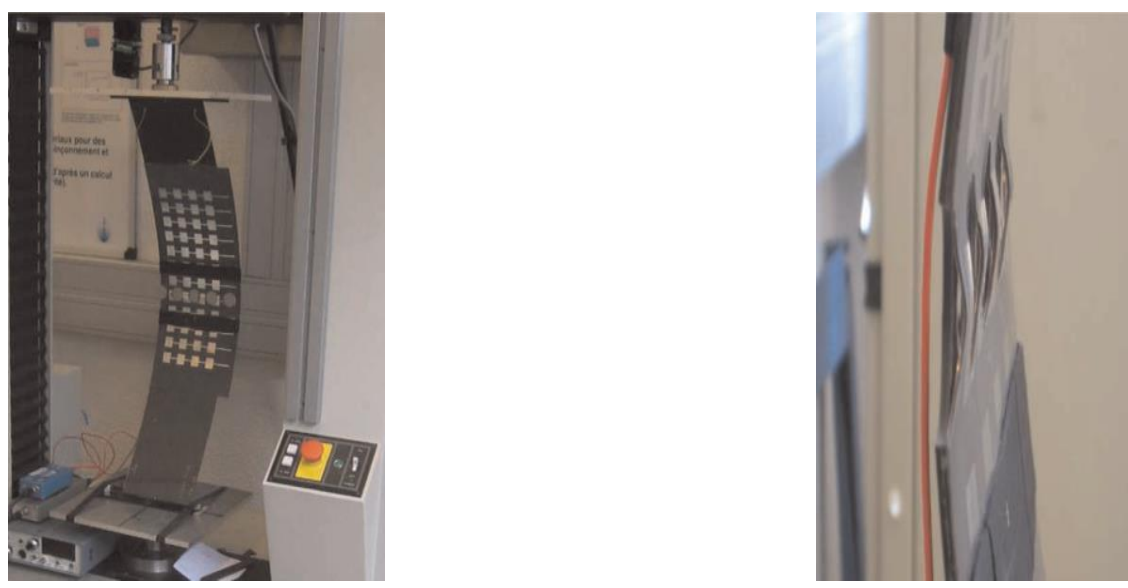

(a) Buckling device

(b) Delamination of the antenna array

Fig. 15 Damaged CLAS 


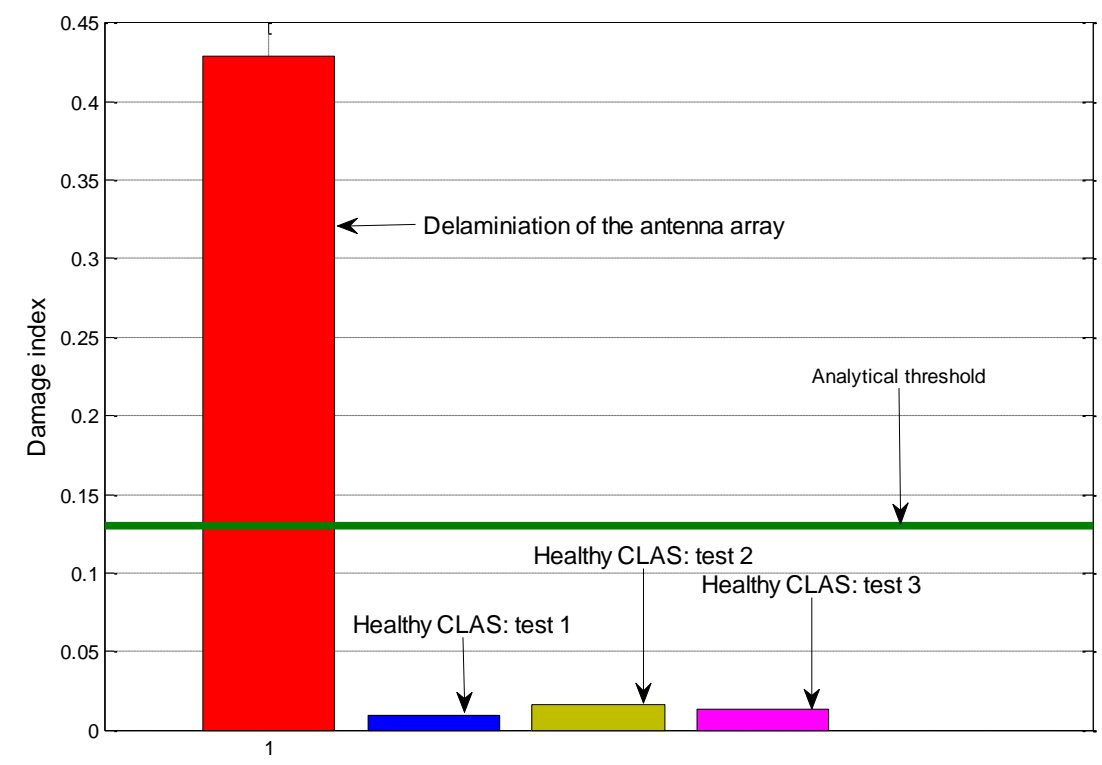

Fig. 16 Results of the damage detection of the CLAS

\section{Discussion}

In baseline subtraction SHM methods, one has to deal with two major issues: (a) building and managing online healthy baseline data that includes the effects of varying environmental and operational conditions, (b) finding a good tradeoff between detectability (the smallest detectable damage) and the rate of false alarm. In the present work, an attempt to address these two issues has been proposed. Indeed, an iterative approach relying on instantaneous baseline measurement is proposed to avoid the use of a presorted database for the healthy state. Then the robustness of the detection is enhanced by elaborating a decisions-making based on an analytical threshold, that: (i) includes the effects of environmental perturbations, (ii) does not require any probabilistic models. Typically, the baseline measurements are recorded when the structure is pristine, they are stored for comparison to future tested data for damage detection purpose following the framework depicted in Fig. 3. When no longer damage is detected, the tested data are used as the new baseline.

One concern with the use of this baseline subtraction method is the fact that the data from a missed damage can be incorporated in the new baseline. This could happen if the damage is small and not severe. The severity and the size of the detected damage depend on the frequency range that we use (higher is the frequency; smallest is the damage to be detected). However, as explained in the introduction, low frequency measurements were our interest.

The proposed damage detection is related to the signal sensors acquired. These signals can be either vibratory or ultrasound data (such as Lamb wave), where their choice is related to the size of damage that we look to detect. In real-world application, the relevant smallest size of damage as the Barely Visible Impact Damage is based on expert point of view of the concerned application. 
Once this smallest size defined, the use of vibratory or ultrasonic data is defined by chosen a welldefined size of sensors/actuators and electronic equipment's. Then, the proposed incremental baseline can be applied.

\section{Conclusions}

In this paper, the problem of output-only vibration based damage detection under changing environmental conditions on MAS structures was studied. Through the independent component analysis method, feature matrices were extracted from the data to characterize the behavior of the studied structures and their models, and a damage index (DI) based on angle between subspaces was proposed.

The originality of the present work was: on one hand to develop a new criterion for the decision- making. The key idea behind was to bind analytically using matrix perturbation theory the disturbances that a healthy monitored structure undergoes, without any probabilistic models. Either in FE simulations and experiments, the proposed approach has shown its robustness regarding damage detection and boundary condition changes. On the other hand, this work has shown the possibility of incorporating different capabilities (transmission/receive function and health monitoring) on a MAS structure.

In the work under progress, experiments on changes in other boundary conditions are underway to consolidate the robustness of the proposed method regarding false-positive alarms.

\section{Acknowledgments}

This work was supported by the project: Smart Materials and Structures for Electromagnetics (SMSE) funded by the consortium Aerospace Paris Région "ASTech". The authors wish to thanks all the persons involved in this project.

\section{References}

Adhikari, S. (2006), "Damping modeling using generalized proportional damping", J. Sound Vib, 293(1-2), 156-170.

Balmes, E and Deraemaeker, A. (2013), "Modeling structures with piezoelectric materials", SDT tutorial, SDTools, Paris, France.

Belouchrani, A., bed-Meraim, K., Cardoso, J. F., and Moulines, E. (1997), "A Blind source separation technique using second-order statistics", IEEE Signal Process, 45(2), 434-444.

Comon, P., and Jutten, C. (2010), Handbook of Blind Source Separation, Elsevier, Burlington, Vermont, USA.

Cover, T. M., and Thomas, J. A. (2006), Elements of Information Theory, John Wiley \& Sons, New Jersey, USA.

De Boe, P., and Golinval, J. C. (2003), "Principal component analysis of a piezosensor array for damage localization", Struct Health Monit, 2(2), 137-144.

Donoho, D. (1981), "On Minimum entropy deconvolution", J. Time Ser. Anal (II), 565-609.

Elseifi, M. A (2010), "Independent component analysis and K-means clustering for damage detection in structural systems", Structural Dynamics and Materials Conference, Orlando, Florida, April. 
Farrar, C. R., and Lieven, N. A. J. (2007), "Damage prognosis: The future of structural health monitoring", Philos. Trans. R. Soc. Lond., A, 365(1851), 623-632.

Ferroperm (2009), "Material data based on typical values for piezoceramic", Paris France.

Gävert, H., Jaakko, H., Särelä, J., and Hyvärinen, A (2005), "The FastICA Matlab package", Aalto, Finland.

Golub, G. H., and Van Loan, C. F. (1983), Matrix Computation, Johns Hopkins University Press, Baltimore, Maryland, USA.

Hajrya, R., and Mechbal, N. (2013), "Principal component analysis and perturbation theory-based robust damage detection of multifunctional aircraft structure", Struct Health Monit, 12(3), 263-277.

Hajrya, R., Mechbal, N., and Vergé, M. (2010), "Active damage detection and localization applied to a composite structure using piezoceramic patches", IEEE Conference on Control and Fault-Tolerant Systems, Nice, October.

Hyvärinen, A., Karhunen, J., and Oja, E. (2001), Independent Component Analysis: Algorithms and Applications, John Wiley \& Sons, New York, NY, USA.

Kerschen, G., Poncelet, F., and Golinval, J. C. (2007), "Physical interpretation of independent component analysis in structural dynamics", Mech Syst and Signal Pr, 21(4), 1561-1575.

Liu, X., Zhou, C., and Jiang, Z. (2012), "Damage localization in plate-like structure using built-in PZT sensor network", Smart Struct Syst, 9(1), 21-33.

Lockyer, A. J., Alt, K. H., Kudva, J. N., Kinslow, R. W., and Goetz, A. C. (1996), "Conformal load-bearing antenna structures (CLAS): Initiative for Multiple Military and Commercial Applications", SPIE Conference on Smart Structures and Materials, San Diego, CA, March.

Mahzan, S., Staszewski, W. J., and Worden, K. (2010), "Experimental studies on impact damage location in composite aerospace structures using genetic algorithms and neural networks", Smart Struct Syst, 6(2),

147-165.

McNeill, S. I., and Zimmerman, D. C. (2008), "A Framework for blind modal identification using joint approximate diagonalization", Mech Syst and Signal Pr, 22(7), 1526-1548.

Mujica, L. E., Rodellar, J., Fernández, A., and Güemes, A. (2011), "Q-Statistic and T2-Statistic PCA-based measures for damage assessment in structures", Struct Health Monit, 10(5), 539-553.

Nguyen, V. H., and Golinval, J. C. (2011), "Damage detection using blind source separation techniques", Conference Proceedings of the Society for Experimental Mechanics Series, Florida, January.

Preumont, P. (2002), Vibration control of active structures-An introduction, (3rd Edition), Springer, New York, NY, USA.

SMSE (2010), "Research Report-SMSE project: smart materials and structures for electromagnetics, Document INE-MSI_DE-0004-A", Paris, France.

Sohn, H., Allen, D. W., Worden, K., and Farrar, C. R. (2005), "Structural damage classification using extreme value statistics", J Dyna Syst-T-ASME, 127(1), 125-132.

Stewart, G. M., and Sun, J. G. (1990), Matrix Perturbation Theory, Academic Press Boston, Massachusetts, USA.

Valliappan, S., Mufti, V., Wohua, Zh. (1990), " Finite element analysis of anisotropic damage mechanics problems", Eng Fract Mech, 35(6), 1061-1071.

Wang, Q., and Wu, N. (2012), "A review on structural enhancement and repair using piezoelectric materials and shape memory alloys", Smart Mater Struct, 21(1), 1-23.

Wedin, P. (1972), "Perturbation bounds in connection with singular value decomposition", Numer Math,12(1), 99-111.

Worden, K., Manson, G., and Fieller, N. R. J. (2000), "Damage detection using outlier analysis", J. Sound Vib, 229(3), 647-667.

Zang, C., Friswell, M. I., and Imregun, M. (2004), "Structural damage detection using independent component analysis", Struct Health Monit, 3(1), 69-83.

Zhou, W., and Chelidze, D. (2007), "Blind source separation based vibration mode identification", Mech Syst and Signal Pr, 21(8), 3072-3087. 